\title{
hglm: A Package for Fitting Hierarchical Generalized Linear Models
}

\author{
by Lars Rönnegård, Xia Shen and Moudud Alam
}

\begin{abstract}
We present the hglm package for fitting hierarchical generalized linear models. It can be used for linear mixed models and generalized linear mixed models with random effects for a variety of links and a variety of distributions for both the outcomes and the random effects. Fixed effects can also be fitted in the dispersion part of the model.
\end{abstract}

\section{Introduction}

The hglm package (Alam et al., 2010) implements the estimation algorithm for hierarchical generalized linear models (HGLM; Lee and Nelder, 1996). The package fits generalized linear models (GLM; McCullagh and Nelder, 1989) with random effects, where the random effect may come from a distribution conjugate to one of the exponential-family distributions (normal, gamma, beta or inverse-gamma). The user may explicitly specify the design matrices both for the fixed and random effects. In consequence, correlated random effects, as well as random regression models can be fitted. The dispersion parameter can also be modeled with fixed effects.

The main function is $\mathrm{hglm}()$ and the input is specified in a similar manner as for $g \operatorname{lm}()$. For instance,

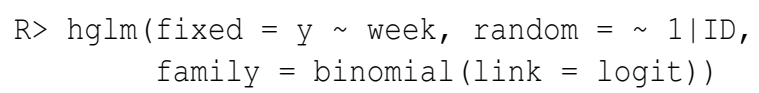

fits a logit model for $y$ with week as fixed effect and ID representing the clusters for a normally distributed random intercept. Given an hglm object, the standard generic functions are print (), summary () and plot ().

Generalized linear mixed models (GLMM) have previously been implemented in several $\mathrm{R}$ functions, such as the lmer() function in the lme4 package (Bates and Maechler, 2010) and the glmmPQL () function in the MASS package (Venables and Ripley, 2002). In GLMM, the random effects are assumed to be Gaussian whereas the hglm() function allows other distributions to be specified for the random effect. The hglm() function also extends the fitting algorithm of the dglm package (Dunn and Smyth, 2009) by including random effects in the linear predictor for the mean, i.e. it extends the algorithm so that it can cope with mixed models. Moreover, the model specification in hglm() can be given as a formula or alternatively in terms of $y, x, z$ and $x$. disp. Here $y$ is the vector of observed responses, $x$ and $\mathrm{Z}$ are the design matrices for the fixed and random effects, respectively, in the linear predictor for the means and $\mathrm{X}$.disp is the design matrix for the fixed effects in the dispersion parameter. This enables a more flexible modeling of the random effects than specifying the model by an $\mathrm{R}$ formula. Consequently, this option is not as user friendly but gives the user the possibility to fit random regression models and random effects with known correlation structure.

The hglm package produces estimates of fixed effects, random effects and variance components as well as their standard errors. In the output it also produces diagnostics such as deviance components and leverages.

\section{Three illustrating models}

The hglm package makes it possible to

1. include fixed effects in a model for the residual variance,

2. fit models where the random effect distribution is not necessarily Gaussian,

3. estimate variance components when we have correlated random effects.

Below we describe three models that can be fitted using $\operatorname{hglm}($ ), which illustrate these three points. Later, in the Examples section, five examples are presented that include the R syntax and output for the $\operatorname{hg} \operatorname{lm}($ ) function.

\section{Linear mixed model with fixed effects in the residual variance}

We start by considering a normal-normal model with heteroscedastic residual variance. In biology, for instance, this is important if we wish to model a random genetic effect (e.g., Rönnegård and Carlborg, 2007) for a trait $y$, where the residual variance differs between the sexes.

For the response $y$ and observation number $i$ we have:

$$
\begin{aligned}
y_{i} \mid \beta, u, \beta_{d} & \sim N\left(X_{i} \beta+Z_{i} u, \exp \left(X_{d, i} \beta_{d}\right)\right) \\
u & \sim \operatorname{MVN}\left(0, \mathbf{I} \sigma_{u}^{2}\right)
\end{aligned}
$$

where $\beta$ are the fixed effects in the mean part of the model, the random effect $u$ represents random variation among clusters of observations and $\beta_{d}$ is the fixed effect in the residual variance part of the model. The variance of the random effect $u$ is given by $\sigma_{u}^{2}$. 
The subscript $i$ for the matrices $\mathbf{X}, \mathbf{Z}$, and $\mathbf{X}_{d}$ indicates the $i^{\prime}$ th row. Here, a log link function is used for the residual variance and the model for the residual variance is therefore given by $\exp \left(X_{d, i} \beta_{d}\right)$. In the more general GLM notation, the residual variance here is described by the dispersion term $\phi$, so we have $\log \left(\phi_{i}\right)=X_{d, i} \beta_{d}$.

This model cannot be fitted with the dglm package, for instance, because we have random effects in the mean part of the model. It is also beyond the scope of the lmer () function since we allow a model for the residual variance.

The implementation in hglm() for this model is demonstrated in Example 2 in the Examples section below.

\section{A Poisson model with gamma distributed random effects}

For dependent count data it is common to model a Poisson distributed response with a gamma distributed random effect (Lee et al., 2006). If we assume no overdispersion conditional on $u$ and thereby have a fixed dispersion term, this model may be specified as:

$$
E\left(y_{i} \mid \beta, u\right)=\exp \left(X_{i} \beta+Z_{i} v\right)
$$

where a level $j$ in the random effect $v$ is given by $v_{j}=\log \left(u_{j}\right)$ and $u_{j}$ are iid with gamma distribution having mean and variance: $E\left(u_{j}\right)=1$, $\operatorname{var}\left(u_{j}\right)=\lambda$.

This model can also be fitted with the hglm package, since it extends existing GLMM functions (e.g. lmer ()) to allow a non-normal distribution for the random effect. Later on, in Example 3, we show the hglm ( ) code used for fitting a gamma-Poisson model with fixed effects included in the dispersion parameter.

\section{A linear mixed model with a correlated random effect}

In animal breeding it is important to estimate variance components prior to ranking of animal performances (Lynch and Walsh, 1998). In such models the genetic effect of each animal is modeled as a level in a random effect and the correlation structure $\mathbf{A}$ is a matrix with known elements calculated from the pedigree information. The model is given by

$$
\begin{aligned}
y_{i} \mid \beta, u & \sim N\left(X_{i} \beta+Z_{i} u, \sigma_{e}^{2}\right) \\
u & \sim \operatorname{MVN}\left(0, \mathbf{A} \sigma_{u}^{2}\right)
\end{aligned}
$$

This may be reformulated as (see Lee et al., 2006; Rönnegård and Carlborg, 2007)

$$
\begin{aligned}
y_{i} \mid \beta, u & \sim N\left(X_{i} \beta+Z_{i}^{*} u^{*}, \sigma_{e}^{2}\right) \\
u^{*} & \sim \operatorname{MVN}\left(0, \mathbf{I} \sigma_{u}^{2}\right)
\end{aligned}
$$

where $\mathbf{Z}^{*}=\mathbf{Z L}$ and $\mathbf{L}$ is the Cholesky factorization of A.

Thus the model can be fitted using the hglm() function with a user-specified input matrix $\mathrm{Z}$ (see $\mathrm{R}$ code in Example 4 below).

\section{Overview of the fitting algorithm}

The fitting algorithm is described in detail in Lee et al. (2006) and is summarized as follows. Let $n$ be the number of observations and $k$ be the number of levels in the random effect. The algorithm is then:

1. Initialize starting values.

2. Construct an augmented model with response $y_{\text {aug }}=\left(\begin{array}{c}y \\ E(u)\end{array}\right)$.

3. Use a GLM to estimate $\beta$ and $v$ given the vector $\phi$ and the dispersion parameter for the random effect $\lambda$. Save the deviance components and leverages from the fitted model.

4. Use a gamma GLM to estimate $\beta_{d}$ from the first $n$ deviance components $d$ and leverages $h$ obtained from the previous model. The response variable and weights for this model are $d /(1-h)$ and $(1-h) / 2$, respectively. Update the dispersion parameter by putting $\phi$ equal to the predicted response values for this model.

5. Use a similar GLM as in Step 4 to estimate $\lambda$ from the last $k$ deviance components and leverages obtained from the GLM in Step 3.

6. Iterate between steps 3-5 until convergence.

For a more detailed description of the algorithm in a particular context, see below.

\section{H-likelihood theory}

Let $y$ be the response and $u$ an unobserved random effect. The hglm package fits a hierarchical model $y \mid u \sim f_{m}(\mu, \phi)$ and $u \sim f_{d}(\psi, \lambda)$ where $f_{m}$ and $f_{d}$ are specified distributions for the mean and dispersion parts of the model.

We follow the notation of Lee and Nelder (1996), which is based on the GLM terminology by McCullagh and Nelder (1989). We also follow the likelihood approach where the model is described in terms of likelihoods. The conditional (log-)likelihood for $y$ given $u$ has the form of a GLM

$$
\ell\left(\theta^{\prime}, \phi ; y \mid u\right)=\frac{y \theta^{\prime}-b\left(\theta^{\prime}\right)}{a(\phi)}+c(y, \phi)
$$

where $\theta^{\prime}$ is the canonical parameter, $\phi$ is the dispersion term, $\mu^{\prime}$ is the conditional mean of $y$ given $u$ 
where $\eta^{\prime}=g\left(\mu^{\prime}\right)$, i.e. $g()$ is a link function for the GLM. The linear predictor is given by $\eta^{\prime}=\eta+v$ where $\eta=X \beta$ and $v=v(u)$ for some strict monotonic function of $u$. The link function $v(u)$ should be specified so that the random effects occur linearly in the linear predictor to ensure meaningful inference from the h-likelihood (Lee et al., 2007). The h-likelihood or hierarchical likelihood is defined by

$$
h=\ell\left(\theta^{\prime}, \phi ; y \mid u\right)+\ell(\alpha ; v)
$$

where $\ell(\alpha ; v)$ is the log density for $v$ with parameter $\alpha$. The estimates of $\beta$ and $v$ are given by $\frac{\partial h}{\partial \beta}=0$ and $\frac{\partial h}{\partial v}=0$. The dispersion components are estimated by maximizing the adjusted profile h-likelihood

$$
h_{p}=\left(h-\frac{1}{2} \log \left|-\frac{1}{2 \pi} H\right|\right)_{\beta=\hat{\beta}, v=\hat{v}}
$$

where $H$ is the Hessian matrix of the h-likelihood. The dispersion term $\phi$ can be connected to a linear predictor $X_{d} \beta_{d}$ given a link function $g_{d}(\cdot)$ with $g_{d}(\phi)=X_{d} \beta_{d}$. The adjusted profile likelihoods of $\ell$ and $h$ may be used for inference of $\beta, v$ and the dispersion parameters $\phi$ and $\lambda$ (pp. 186 in Lee et al., 2006). More detail and discussion of h-likelihood theory is presented in the hglm vignette.

\section{Detailed description of the hglm fitting al- gorithm for a linear mixed model with het- eroscedastic residual variance}

In this section we describe the fitting algorithm in detail for a linear mixed model where fixed effects are included in the model for the residual variance. The extension to distributions other than Gaussian is described at the end of the section.

Lee and Nelder (1996) showed that linear mixed models can be fitted using a hierarchy of GLM by using an augmented linear model. The linear mixed model

$$
\begin{aligned}
& y=\mathbf{X} b+\mathbf{Z} u+e \\
& v=\mathbf{Z Z}^{T} \sigma_{u}^{2}+\mathbf{R} \sigma_{e}^{2}
\end{aligned}
$$

where $\mathbf{R}$ is a diagonal matrix with elements given by the estimated dispersion model (i.e. $\phi$ defined below). In the first iteration of the HGLM algorithm, $\mathbf{R}$ is an identity matrix. The model may be written as an augmented weighted linear model:

$$
y_{a}=\mathbf{T}_{a} \delta+e_{a}
$$

where

$$
\begin{aligned}
y_{a} & =\left(\begin{array}{l}
y \\
0_{q}
\end{array}\right) & \mathbf{T}_{a} & =\left(\begin{array}{cc}
\mathbf{X} & \mathbf{Z} \\
\mathbf{0} & \mathbf{I}_{q}
\end{array}\right) \\
\delta & =\left(\begin{array}{l}
b \\
u
\end{array}\right) & e_{a} & =\left(\begin{array}{c}
e \\
-u
\end{array}\right)
\end{aligned}
$$

Here, $q$ is the number of columns in $\mathbf{Z}, 0_{q}$ is a vector of zeros of length $q$, and $\mathbf{I}_{q}$ is the identity matrix of size $q \times q$. The variance-covariance matrix of the augmented residual vector is given by

$$
V\left(e_{a}\right)=\left(\begin{array}{cc}
\mathbf{R} \sigma_{e}^{2} & \mathbf{0} \\
\mathbf{0} & \mathbf{I}_{q} \sigma_{u}^{2}
\end{array}\right)
$$

Given $\sigma_{e}^{2}$ and $\sigma_{u}^{2}$, this weighted linear model gives the same estimates of the fixed and random effects ( $b$ and $u$ respectively) as Henderson's mixed model equations (Henderson, 1976).

The estimates from weighted least squares are given by:

$$
\mathbf{T}_{a}^{t} \mathbf{W}^{-1} \mathbf{T}_{a} \hat{\delta}=\mathbf{T}_{a}^{t} \mathbf{W}^{-1} y_{a}
$$

where $\mathbf{W} \equiv V\left(e_{a}\right)$.

The two variance components are estimated iteratively by applying a gamma GLM to the residuals $e_{i}^{2}$ and $u_{i}^{2}$ with intercept terms included in the linear predictors. The leverages $h_{i}$ for these models are calculated from the diagonal elements of the hat matrix:

$$
\mathbf{H}_{a}=\mathbf{T}_{a}\left(\mathbf{T}_{a}^{t} \mathbf{W}^{-1} \mathbf{T}_{a}\right)^{-1} \mathbf{T}_{a}^{t} \mathbf{W}^{-1}
$$

A gamma GLM is used to fit the dispersion part of the model with response

$$
y_{d, i}=e_{i}^{2} /\left(1-h_{i}\right)
$$

where $E\left(y_{d}\right)=\mu_{d}$ and $\mu_{d} \equiv \phi$ (i.e. $\sigma_{e}^{2}$ for a Gaussian reponse). The GLM model for the dispersion parameter is then specified by the link function $g_{d}($. and the linear predictor $X_{d} \beta_{d}$, with prior weights $\left(1-h_{i}\right) / 2$, for

$$
g_{d}\left(\mu_{d}\right)=X_{d} \beta_{d}
$$

Similarly, a gamma GLM is fitted to the dispersion term $\alpha$ (i.e. $\sigma_{u}^{2}$ for a GLMM) for the random effect $v$, with

$$
y_{\alpha, j}=u_{j}^{2} /\left(1-h_{n+j}\right), j=1,2, \ldots, q
$$

and

$$
g_{\alpha}\left(\mu_{\alpha}\right)=\lambda
$$

where the prior weights are $\left(1-h_{n+j}\right) / 2$ and the estimated dispersion term for the random effect is given by $\hat{\alpha}=g_{\alpha}^{-1}(\hat{\lambda})$.

The algorithm iterates by updating both $\mathbf{R}=$ $\operatorname{diag}(\hat{\phi})$ and $\sigma_{u}^{2}=\hat{\alpha}$, and subsequently going back to Eq. (4).

For a non-Gaussian response variable $y$, the estimates are obtained simply by fitting a GLM instead of Eq. (4) and by replacing $e_{i}^{2}$ and $u_{j}^{2}$ with the deviance components from the augmented model (see Lee et al., 2006). 


\section{Implementation details}

\section{Distributions and link functions}

There are two important classes of models that can be fitted in hglm: GLMM and conjugate HGLM. GLMMs have Gaussian random effects. Conjugate HGLMs have been commonly used partly due to the fact that explicit formulas for the marginal likelihood exist. HGLMs may be used to fit models in survival analysis (frailty models), where for instance the complementary-log-log link function can be used on binary responses (see e.g., Carling et al., 2004). The gamma distribution plays an important role in modeling responses with a constant coefficient of variation (see Chapter 8 in McCullagh and Nelder, 1989). For such responses with a gamma distributed random effect we have a gamma-gamma model. A summary of the most important models is given in Tables 1 and 2 . Note that the random-effect distribution can be an arbitrary conjugate exponential-family distribution. For the specific case where the random-effect distribution is a conjugate to the distribution of $y$, this is called a conjugate HGLM. Further implementation details can be found in the hglm vignette.

\section{Possible future developments}

In the current version of hglm() it is possible to include a single random effect in the mean part of the model. An important development would be to include several random effects in the mean part of the model and also to include random effects in the dispersion parts of the model. The latter class of models is called Double HGLM and has been shown to be a useful tool for modeling heavy tailed distributions (Lee and Nelder, 2006).

The algorithm of hglm ( ) gives true marginal likelihood estimates for the fixed effects in conjugate HGLM (Lee and Nelder, 1996, pp. 629), whereas for other models the estimates are approximated. Lee and co-workers (see Lee et al., 2006, and references therein) have developed higher-order approximations, which are not implemented in the current version of the hglm package. For such extensions, we refer to the commercially available GenStat software (Payne et al., 2007), the recently available $R$ package HGLMMM (Molas, 2010) and also to coming updates of hglm.

\section{Examples}

\section{Example 1: A linear mixed model}

Data description The output from the hglm () function for a linear mixed model is compared to the results from the lme () function in the nlme (Pinheiro et al., 2009) package using simulated data. In the simulated data there are five clusters with 20 observa- tions in each cluster. For the mean part of the model, the simulated intercept value is $\mu=0$, the variance for the random effect is $\sigma_{u}^{2}=0.2$, and the residual variance is $\sigma_{e}^{2}=1.0$.

Both functions produce the same estimate of the fixed intercept effect of 0.1473 (s.e. 0.16) and also the same variance component estimates. The summary.hglm() function gives the estimate of the variance component for the random intercept (0.082) as well as the residual variance (0.84). It also gives the logarithm of the variance component estimates together with standard errors below the lines Model estimates for the dispersion term and Dispersion model for the random effects. The lme() function gives the square root of the variance component estimates.

The model diagnostics produced by the plot.hglm function are shown in Figures 1 and 2. The data are completely balanced and therefore produce equal leverages (hatvalues) for all observations and also for all random effects (Figure 1). Moreover, the assumption of the deviance components being gamma distributed is acceptable (Figure 2).

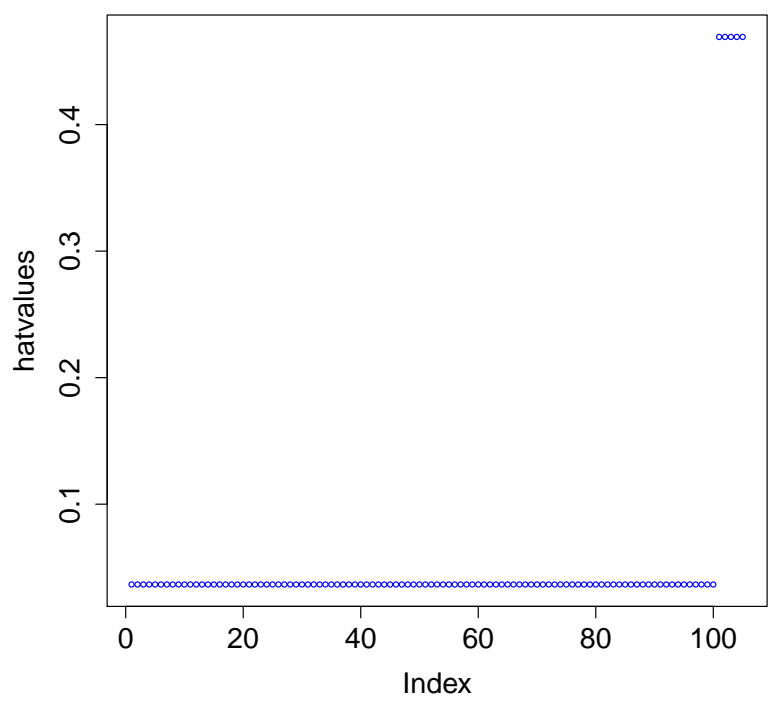

Figure 1: Hatvalues (i.e. diagonal elements of the augmented hat-matrix) for each observation 1 to 100 , and for each level in the random effect (index 101105). 
Table 1: Commonly used distributions and link functions possible to fit with hglm ()

\begin{tabular}{lllll}
\hline Model name & $y \mid u$ distribution & Link $g(\mu)$ & $u$ distribution & Link $v(u)$ \\
\hline Linear mixed model & Gaussian & identity & Gaussian & identity \\
Binomial conjugate & Binomial & logit & Beta & logit \\
Binomial GLMM & Binomial & logit & Gaussian & identity \\
Binomial frailty & Binomial & comp-log-log & Gamma & $\log$ \\
Poisson GLMM & Poisson & $\log$ & Gaussian & identity \\
Poisson conjugate & Poisson & $\log$ & Gamma & log \\
Gamma GLMM & Gamma & $\log$ & Gaussian & identity \\
Gamma conjugate & Gamma & inverse & Inverse-Gamma & inverse \\
Gamma-Gamma & Gamma & $\log$ & Gamma & log \\
\hline
\end{tabular}

Table 2: hglm code for commonly used models

\begin{tabular}{|c|c|c|}
\hline Model name & Setting for family argument & Setting for rand. family argument \\
\hline Linear mixed model $^{a}$ & gaussian (link $=$ identity) & gaussian (link = identity) \\
\hline Beta-Binomial & binomial (link = logit) & $\operatorname{Beta}(\operatorname{link}=\operatorname{logit})$ \\
\hline Binomial GLMM & binomial (link = logit) & gaussian(link = identity) \\
\hline Binomial frailty & binomial (link = cloglog) & Gamma $($ link $=\log )$ \\
\hline Poisson GLMM & poisson (link $=\log )$ & gaussian (link = identity) \\
\hline Poisson frailty & poisson (link = log) & Gamma $($ link $=$ log $)$ \\
\hline Gamma GLMM & Gamma $($ link $=$ log $)$ & gaussian (link = identity) \\
\hline Gamma conjugate & Gamma (link = inverse) & inverse.gamma (link = inverse) \\
\hline Gamma-Gamma & Gamma $($ link $=$ log $)$ & Gamma (link $=$ log) \\
\hline
\end{tabular}

${ }^{a}$ For example, the hglm ( ) code for a linear mixed model is

hglm (family $=$ gaussian $(l i n k=$ identity), rand.family $=\operatorname{gaussian}($ link $=$ identity $), \ldots$ )
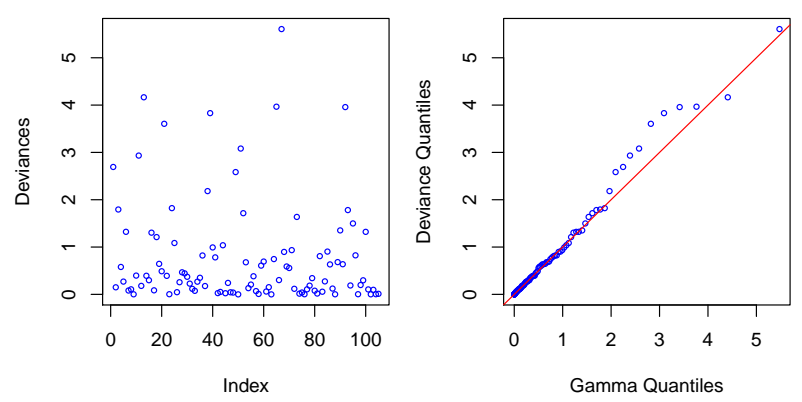

Figure 2: Deviance diagnostics for each observation and each level in the random effect. lows:

The R code and output for this example is as fol-

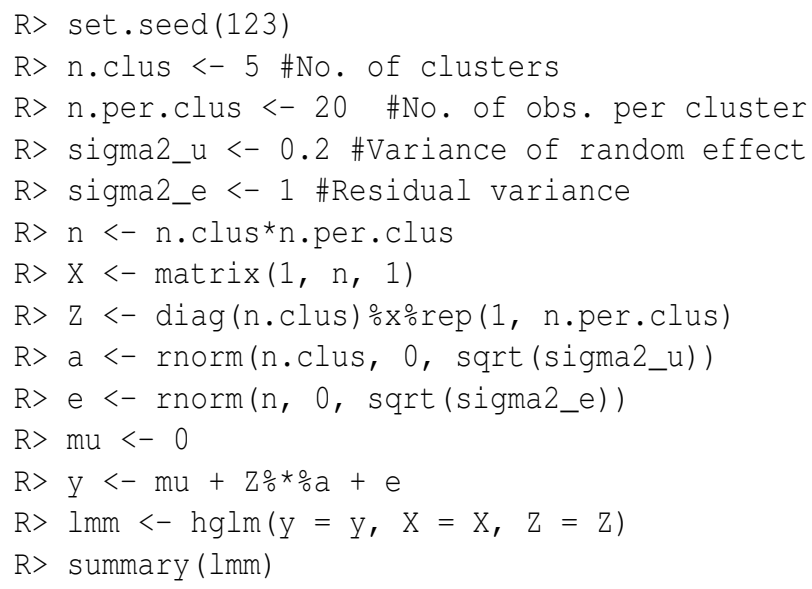

Call:

hglm.default $(x=x, y=y, z=z)$

DISPERSION MODEL

WARNING: h-likelihood estimates through EQL can be biased. Model estimates for the dispersion term:[1] 0.8400608

Model estimates for the dispersion term:

Link $=\log$

Effects:

Estimate Std. Error

$-0.1743 \quad 0.1441$

Dispersion $=1$ is used in Gamma model on deviances to calculate the standard error(s).

Dispersion parameter for the random effects

[1] 0.08211

Dispersion model for the random effects:

Link $=\log$

Effects:

Estimate Std. Error

$-2.4997 \quad 0.8682$

Dispersion $=1$ is used in Gamma model on deviances to calculate the standard error(s).

MEAN MODEL

Summary of the fixed effects estimates Estimate Std. Error $t$ value $\operatorname{Pr}(>|t|)$

$\begin{array}{lllll}X .1 & 0.1473 & 0.1580 & 0.933 & 0.353\end{array}$

Note: P-values are based on 96 degrees of freedom Summary of the random effects estimate Estimate Std. Error

$[2]-,0.0383 \quad 0.1971$ $\left[\begin{array}{lll}{[1,]} & -0.3237 & 0.1971\end{array}\right.$ 


$\begin{array}{rrr}{[3,]} & 0.3108 & 0.1971 \\ {[4,]} & -0.0572 & 0.1971 \\ {[5,]} & 0.1084 & 0.1971\end{array}$

EQL estimation converged in 5 iterations.

R> \#Same analysis with the lme function

R> library (nlme)

R> clus <- rep (1:n.clus,

$+\quad \operatorname{rep}(n \cdot p e r . c l u s$, n.clus ))

$\mathrm{R}>\operatorname{summary}(\operatorname{lme}(\mathrm{y} \sim 0+\mathrm{X}$,

$+\quad$ random $=\sim 1 \mid$ clus $)$ )

Linear mixed-effects model fit by REML

Data: NULL

AIC BIC logLik

$278.635286 .4203-136.3175$

Random effects:

Formula: $\sim 1$ | clus

(Intercept) Residual

StdDev: $0.2859608 \quad 0.9166$

Fixed effects: $\mathrm{y} \sim 0+\mathrm{X}$

Value Std.Error DF t-value p-value

$\begin{array}{llllll}X & 0.1473009 & 0.1573412 & 95 & 0.9361873 & 0.3516\end{array}$

Standardized Within-Group Residuals:

$\begin{array}{rrrrr}\text { Min } & \text { Q1 } & \text { Med } & \text { Q3 } & \text { Max } \\ -2.5834807 & -0.6570612 & 0.0270673 & 0.6677986 & 2.1724148\end{array}$

Number of Observations: 100

Number of Groups: 5

\section{Example 2: Analysis of simulated data for a linear mixed model with heteroscedastic residual variance}

Data description Here, a heteroscedastic residual variance is added to the simulated data from the previous example. Given the explanatory variable $x_{d}$, the simulated residual variance is 1.0 for $x_{d}=0$ and 2.72 for $x_{d}=1$. The output shows that the variance of the random effect is 0.109 , and that $\hat{\beta}_{d}=$ $(-0.32,1.47)$, i.e. the two residual variances are estimated as 0.72 and 3.16. (Code continued from Example 1)

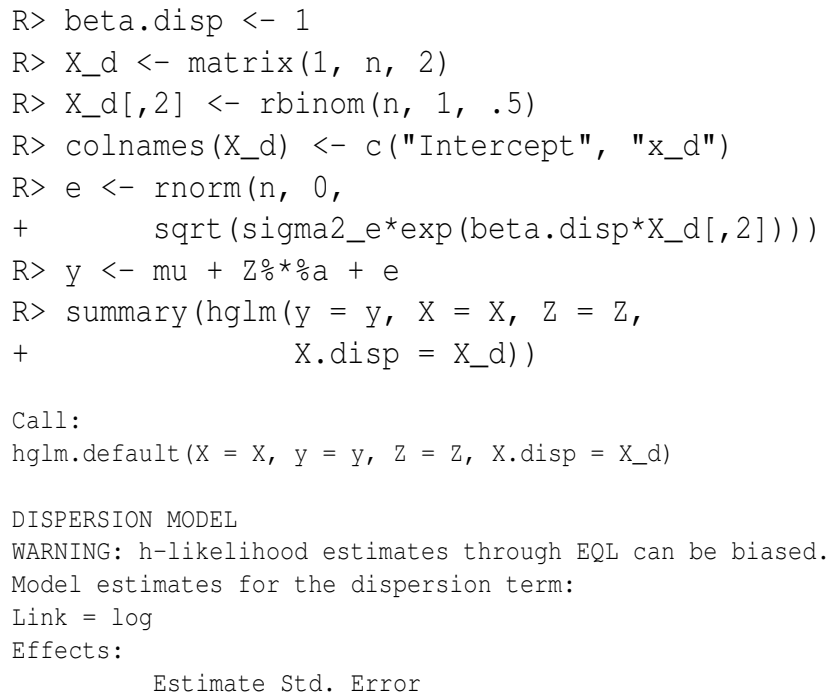

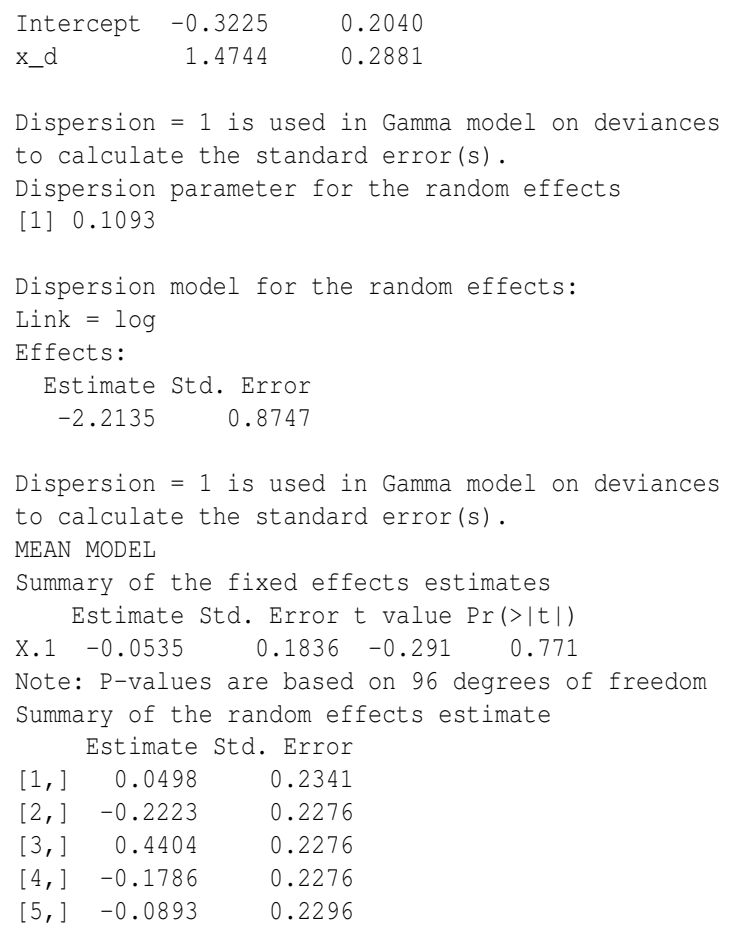

EQL estimation converged in 5 iterations.

Example 3: Fitting a Poisson model with gamma random effects, and fixed effects in the dispersion term

Data description We simulate a Poisson model with random effects and estimate the parameter in the dispersion term for an explanatory variable $x_{d}$. The estimated dispersion parameter for the random effects is 0.6556. (Code continued from Example 2)

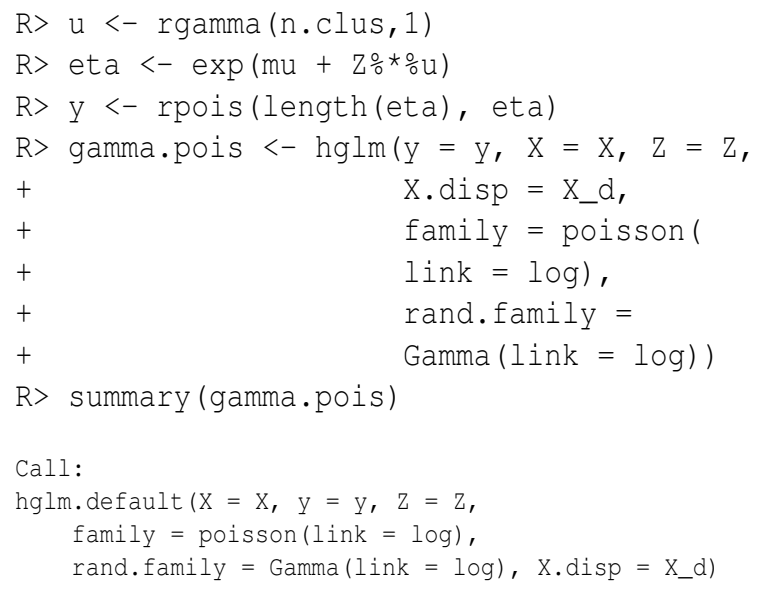

WARNING: h-likelihood estimates through EQL can be biased. Model estimates for the dispersion term:

Link $=\log$

Effects:

$\begin{array}{lrr} & \text { Estimate Std. Error } \\ \text { Intercept } & -0.0186 & 0.2042 \\ \text { x_d } & 0.4087 & 0.2902\end{array}$

Dispersion $=1$ is used in Gamma model on deviances to calculate the standard error(s).

Dispersion parameter for the random effects 


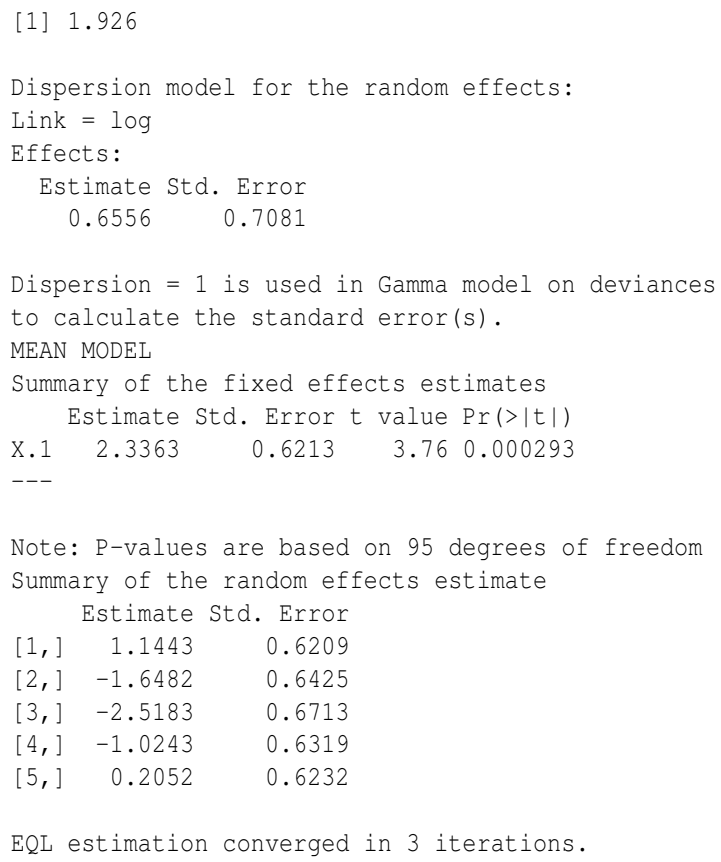

\section{Example 4: Incorporating correlated ran- dom effects in a linear mixed model - a ge- netics example}

Data description The data consists of 2025 individuals from two generations where 1000 individuals have observed trait values $y$ that are approximately normal (Figure 3). The data we analyze was simulated for the QTLMAS 2009 Workshop (Coster et al., 2010) ${ }^{1}$. A longitudinal growth trait was simulated. For simplicity we analyze only the values given on the third occasion at age 265 days.
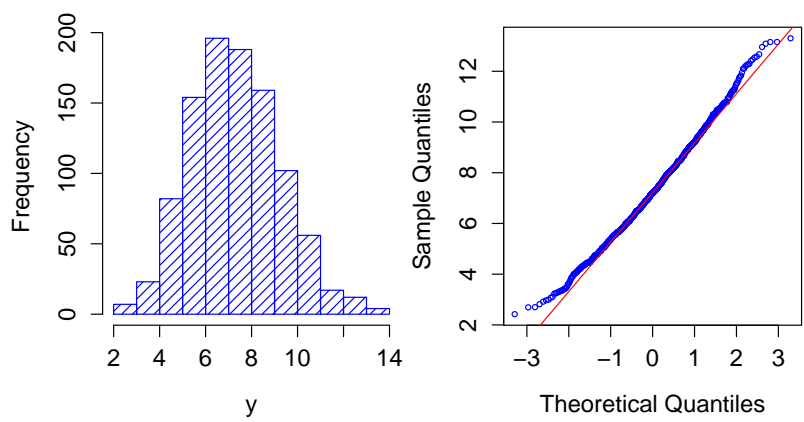

Figure 3: Histogram and qqplot for the analyzed trait.

We fitted a model with a fixed intercept and a random animal effect, $a$, where the correlation structure of $a$ is given by the additive relationhip matrix A (which is obtained from the available pedigree information). An incidence matrix $\mathbf{Z}_{0}$ was constructed and relates observation number with id-number in the pedigree. For observation $y_{i}$ coming from indi- vidual $j$ in the ordered pedigree file $\mathbf{Z}_{0}[i, j]=1$, and all other elements are 0 . Let $\mathbf{L}$ be the Cholesky factorization of $\mathbf{A}$, and $\mathbf{Z}=\mathbf{Z}_{0} \mathbf{L}$. The design matrix for the fixed effects, $\mathbf{X}$, is a column of ones. The estimated variance components are $\hat{\sigma}_{e}^{2}=2.21$ and $\hat{\sigma}_{u}^{2}=1.50$.

The $\mathrm{R}$ code for this example is given below.

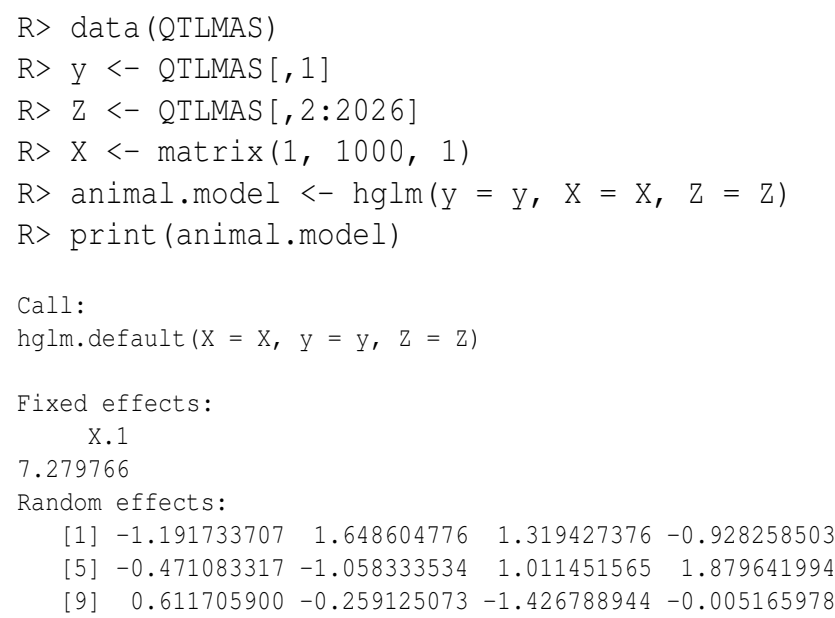

Dispersion parameter for the mean model:[1] 2.211169 Dispersion parameter for the random effects: [1] 1.502516

EQL estimation converged in 2 iterations

\section{Example 5: Binomial-beta model applied to seed germination data}

Data description The seed germination data presented by Crowder (1978) has previously been analyzed using a binomial GLMM (Breslow and Clayton, 1993) and a binomial-beta HGLM (Lee and Nelder, 1996). The data consists of 831 observations from 21 germination plates. The effect of seed variety and type of root extract was studied in a $2 \times 2$ factorial lay-out. We fit the binomial-beta HGLM used by Lee and Nelder (1996) and setting fix.disp $=1 \mathrm{in} \operatorname{hglm}()$ produces comparable estimates to the ones obtained by Lee and Nelder (with differences $<2 \times 10^{-3}$ ). The beta distribution parameter $\alpha$ in Lee and Nelder (1996) was defined as $1 /(2 a)$ where $a$ is the dispersion term obtained from $\operatorname{hglm}()$. The output from the $\mathrm{R}$ code given below gives $\hat{a}=0.0248$ and the corresponding estimate given in Lee and Nelder (1996) is $\hat{a}=1 /(2 \hat{\alpha})=0.023$. We conclude that the hglm package produces similar results as the ones presented in Lee and Nelder (1996) and the dispersion parameters estimated using the EQL method in GenStat differ by less than 1\%. Additional examples, together with comparisons to estimates produced by GenStat, are given in the hglm vignette included in the package on CRAN.

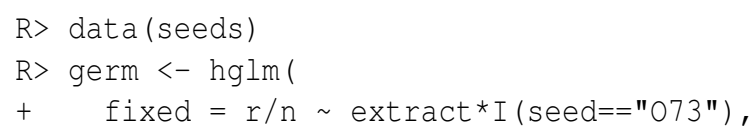

\footnotetext{
${ }^{1}$ http://www.qtlmas2009. wur.nl/UK/Dataset
} 


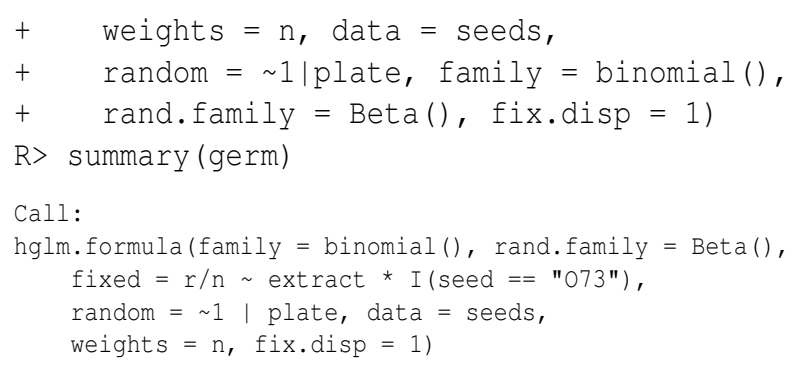

DISPERSION MODEL

WARNING: h-likelihood estimates through EQL can be biased. Model estimates for the dispersion term:[1] 1

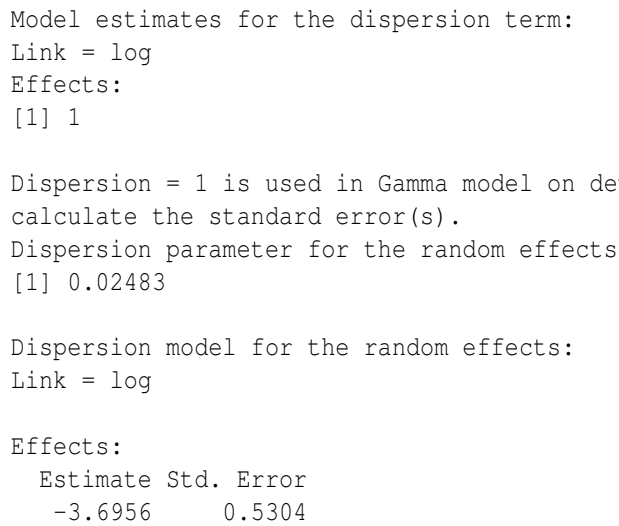

\section{Summary}

The hierarchical generalized linear model approach offers new possibilities to fit generalized linear models with random effects. The hglm package extends existing GLMM fitting algorithms to include fixed effects in a model for the residual variance, fits models where the random effect distribution is not necessarily Gaussian and estimates variance components for correlated random effects. For such models there are important applications in, for instance: genetics (Noh et al., 2006), survival analysis (Ha and Lee,
2005), credit risk modeling (Alam and Carling, 2008), count data (Lee et al., 2006) and dichotomous responses (Noh and Lee, 2007). We therefore expect that this new package will be of use for applied statisticians in several different fields.

\section{Bibliography}

M. Alam and K. Carling. Computationally feasible estimation of the covariance structure in generalized linear mixed models GLMM. Journal of Statistical Computation and Simulation, 78:1227-1237, 2008.

M. Alam, L. Ronnegard, and X. Shen. hglm: Hierarchical Generalized Linear Models, 2010. URL http: //CRAN.R-project.org/package=hglm. R package version 1.1.1.

D. Bates and M. Maechler. Ime4: Linear mixed-effects models using S4 classes, 2010. URL http://CRAN. $\mathrm{R}$-project.org/package=lme 4 . R package version 0.999375-37.

N. E. Breslow and D. G. Clayton. Approximate inference in generalized linear mixed models. Journal of the American Statistical Association, 88:9-25, 1993.

K. Carling, L. Rönnegård, and K. Roszbach. An analysis of portfolio credit risk when counterparties are interdependent within industries. Sveriges Riksbank Working Paper, 168, 2004.

A. Coster, J. Bastiaansen, M. Calus, C. Maliepaard, and M. Bink. QTLMAS 2010: Simulated dataset. BMC Proceedings, 4(Suppl 1):S3, 2010.

M. J. Crowder. Beta-binomial ANOVA for proportions. Applied Statistics, 27:34-37, 1978.

P. K. Dunn and G. K. Smyth. dglm: Double generalized linear models, 2009. URL http: //CRAN. R-project. org/package=dglm. R package version 1.6.1.

I. D. Ha and Y. Lee. Comparison of hierarchical likelihood versus orthodox best linear unbiased predictor approaches for frailty models. Biometrika, 92: 717-723, 2005.

C. R. Henderson. A simple method for computing the inverse of a numerator relationship matrix used in prediction of breeding values. Biometrics, 32(1):69-83, 1976.

Y. Lee and J. A. Nelder. Double hierarchical generalized linear models with discussion. Applied Statistics, 55:139-185, 2006.

Y. Lee and J. A. Nelder. Hierarchical generalized linear models with discussion. J. R. Statist. Soc. B, 58: 619-678, 1996. 
Y. Lee, J. A. Nelder, and Y. Pawitan. Generalized linear models with random effects. Chapman \& Hall/CRC, 2006.

Y. Lee, J. A. Nelder, and M. Noh. H-likelihood: problems and solutions. Statistics and Computing, 17: 49-55, 2007.

M. Lynch and B. Walsh. Genetics and analysis of Quantitative Traits. Sinauer Associates, Inc., 1998. ISBN 087893481.

P. McCullagh and J. A. Nelder. Generalized linear models. Chapman \& Hall/CRC, 1989.

M. Molas. HGLMMM: Hierarchical Generalized Linear Models, 2010. URL http://CRAN.R-project.org/ package=HGLMMM. $R$ package version 0.1.1.

M. Noh and Y. Lee. REML estimation for binary data in GLMMs. Journal of Multivariate Analysis, 98:896915, 2007.

M. Noh, B. Yip, Y. Lee, and Y. Pawitan. Multicomponent variance estimation for binary traits in familybased studies. Genetic Epidemiology, 30:37-47, 2006.

R. W. Payne, D. A. Murray, S. A. Harding, D. B. Baird, and D. M. Soutar. GenStat for Windows (10th edition) introduction, 2007. URL http://www.vsni. co.uk/software/genstat.

J. Pinheiro, D. Bates, S. DebRoy, D. Sarkar, and the $R$ Core team. nlme: Linear and Nonlinear Mixed Effects Models, 2009. URL http://CRAN.R-project. org/package=nlme. R package version 3.1-96.
L. Rönnegård and Ö. Carlborg. Separation of base allele and sampling term effects gives new insights in variance component QTL analysis. BMC Genetics, 8(1), 2007.

W. N. Venables and B. D. Ripley. Modern Applied Statistics with $S$. Springer, New York, fourth edition, 2002. URL http://www.stats.ox.ac.uk/ $\mathrm{pub} / \mathrm{MASS} 4$. ISBN 0-387-95457-0.

Lars Rönnegård

Statistics Unit

Dalarna University, Sweden

and

Department of Animal Breeding and Genetics

Swedish University of Agricultural Sciences, Sweden

Irnedu.se

Xia Shen

Department of Cell and Molecular Biology

Uppsala University, Sweden

and

Statistics Unit

Dalarna University, Sweden

xia.shen@lcb.uu.se

Moudud Alam

Statistics Unit

Dalarna University, Sweden

maaldu.se 